\title{
Shadow Elimination and Blinding Light Suppression for Interactive Projected Displays
}

\author{
Jay Summet, Member, IEEE, Matthew Flagg, Member, IEEE, Tat-Jen Cham, Mem- \\ ber, IEEE, James M. Rehg, Member, IEEE, Rahul Sukthankar, Member, IEEE
}

\begin{abstract}
A major problem with interactive displays based on front-projection is that users cast undesirable shadows on the display surface. This situation is only partially-addressed by mounting a single projector at an extreme angle and warping the projected image to undo keystoning distortions. This paper demonstrates that shadows can be muted by redundantly-illuminating the display surface using multiple projectors, all mounted at different locations. However, this technique alone does not eliminate shadows: multiple projectors create multiple dark regions on the surface (penumbral occlusions) and cast undesirable light onto the users. These problems can be solved by eliminating shadows and suppressing the light that falls on occluding users by actively modifying the projected output. This paper categorizes various methods that can be used to achieve redundant illumination, shadow elimination, and blinding light suppression, and evaluates their performance.
\end{abstract}

Index Terms - Shadow elimination, blinding light suppression, projector calibration, multiple projector display

\section{INTRODUCTION}

The increasing affordability of high quality projectors has allowed the development of multiprojector systems for video walls $[1,2,3,4,5]$ and immersive 3-D virtual environments [6]. In most of these systems, cameras are used to coordinate the aggregation of multiple projectors into a single, large projected display. In constructing a video wall, for example, the geometric alignment of overlapping

Jay Summet, Matthew Flagg, \& James M. Rehg are with the GVU Center \& College of Computing; Georgia Institute of Technology. Tat-Jen Cham is with the School of Computer Engineering; Nanyang Technological University. Rahul Sukthankar is with Intel Research Pittsburgh \& The Robotics Institute; Carnegie Mellon University. projector outputs can be accomplished by using a camera to measure the keystone distortions in projected test patterns and then appropriately prewarping the projected images.

While scalable projector-based displays are quite attractive for visualization applications, interacting with a projected light display currently involves some unpleasant trade-offs. Rear-projection systems support seamless interaction (e.g. the Xerox Liveboard [7], Smart Technologies SmartBoard [8] or Stanford Interactive Mural [9] ), but can be prohibitively expensive and difficult to deploy because of the need for custom installation of a large display screen and the significant space required behind the screen. ${ }^{1}$

Non-projected display technologies (such as LCD and plasma display panels) can be used to produce small to medium sized displays, but become prohibitively expensive as the display size increases. Additionally, front-projected displays offer two novel advantages over rear-projected and nonprojected display technologies: First, they can be moved or steered (via mobile projectors or mirrors) to provide multiple asynchronous displays at several different location in the environment [11]. Second, they can be used to augment objects in the environment, for applications ranging from retail sales to projector guided painting $[12,13]$.

Using front-projection reduces the system's overall cost, increases portability, and allows easier retro-fitting of existing spaces, but suffers from two problems when a user (or inanimate object) moves between the projectors and the display surface: (1) shadows are cast on the display surface; and (2) computer graphics and bright light are projected on the user, which is often a source of distraction for the audience and discomfort for the user.

\footnotetext{
${ }^{1}$ The average cost of office space in the USA is $\$ 77$ per square foot [10].
} 
In this paper we examine five front-projection systems and demonstrate that these problems can be solved without accurate 3-D localization of projectors, cameras, or occluders. We discuss multiple methods we have developed to mitigate and eliminate shadows as well as blinding light. We present a range of systems and algorithms with different hardware and processing requirements that vary from simple passive solutions to fully reactive systems.

We begin with Warped Front Projection (WFP), a single projector passive solutions which move shadows to the side of users, allowing them to interact with graphics directly before them on an interactive surface. Passive Virtual Rear Projection (PVRP) adds a second projector calibrated to overlap with the first, which adds redundant illumination to fill in shadows. Other than some initial calibration, these passive solutions require minimal additional computation while running, but have the drawback that they project blinding light onto users and do not eliminate shadows completely.

By observing the screen with a camera, Active Virtual Rear Projection (AVRP) can detect shadows cast by users and correct for them by boosting the illumination levels, but still casts blinding light on users. Active Virtual Rear Projection with Blinding Light Suppression (AVRP-BLS) detects when projectors are shining on users and eliminates blinding light in addition to shadows. The final system we present, Switching, reduces the computational complexity of the AVRP-BLS system and a version implemented on the Graphics Processing Unit (GPU) allows for real-time operation. In addition to the techniques and algorithms, we present an empirical evaluation of the differing systems' relative performance, and give adoption guidelines for different system needs.

The techniques presented in this paper are particularly useful for projecting interactive surfaces at various locations throughout an environment from steerable projectors. Our solutions provide robustness in the face of occlusions which will be especially important for steered or reconfigurable projected displays, as these projectors may not always be positioned in an optimal position with respect to the display surface.

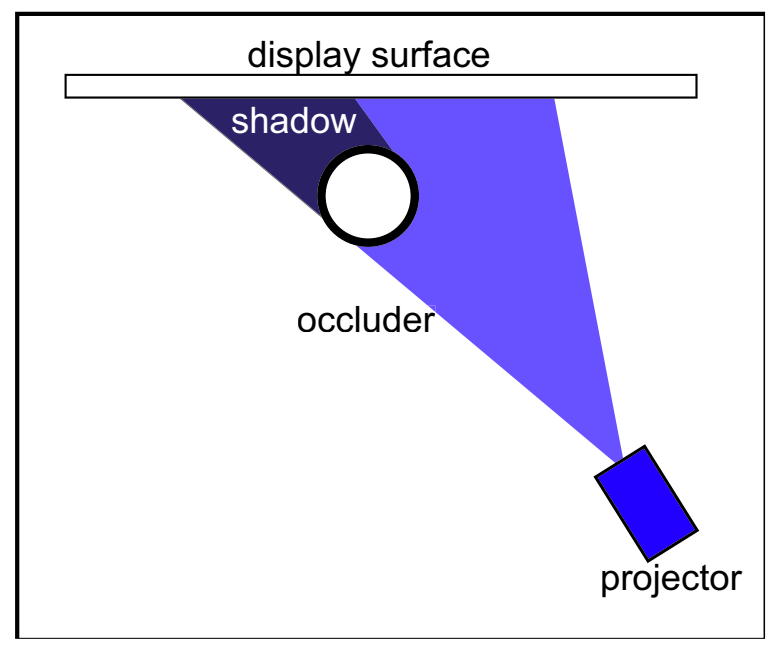

Fig. 1. Warped Front Projection.

\section{WARPED FRONT-PROJECTION}

The simplest method to minimize shadows on the display surface and reduce the amount of blinding light being cast on users is to move the projector to a position near the display surface, and project at an extreme acute angle. An obliquely-mounted projector can have two benefits: (1) it moves the location of the light beam so that the user is less likely to be in it; (2) it may shift the shadow off of the important areas of the display surface. A standard data projector can be mounted at a moderately acute angle, and commodity 3-D video cards can be used to pre-warp the projected image to compensate for keystone distortions, but the projector must remain positioned such that all portions of the display remain within its field of focus.

Commercial projectors designed to be mounted within $1 \mathrm{~m}(3 \mathrm{ft})$ of the display surface use specialized optics such as aspheric mirrors to warp the projected image $[14,15]$. In addition to warping the projected image to compensate for keystone distortions, these optics also have appropriately varying focal lengths for the varying lengths of the beam path.

Even with a very acute projection angle provided by expensive optics, these warped front-projection systems suffer from occlusions whenever the user comes close to or touches the display, making them unsuitable for interactive applications. The areas of occlusion can be filled-in by using a second projector to provide redundant illumination. 


\section{Passive Virtual Rear Projection}

By adding more projectors it is possible to create a display that is more robust to occlusions. We use the general term Virtual Rear Projection to describe the class of display systems which use multiple redundant front projectors to approximate the experience of a rear projected surface. Most areas that are shadowed in one projector can be illuminated by a redundant projector with an unoccluded view. Shadows resulting from all of the projectors being occluded are termed umbral, and those where at least one projector is not occluded are termed penumbral. By definition, the system cannot control lighting within an umbra, so we strive to avoid umbral occlusions by positioning the projectors so that the display is illuminated from several different directions. The largest challenge to providing passive redundant illumination (See Figure 2) is for the system to accurately align the projected images on the display surface. Homographies can be used to align the projected images to within sub-pixel accuracy.

\section{A. Homographies for Calibration of Projector- Camera Systems}

In a multi-projector system, several projectors are positioned so that their outputs converge onto a display surface (see Figure 2). The goal is to combine the light from the projectors to create a single, sharp image on the surface. Clearly, one cannot simply project the same raw image simultaneously through the different projectors; not only does a given point on the surface correspond to very different pixel locations in each projector, but the image produced on the surface from any single projector will be distorted as the projectors are mounted off-center.

We assume that the positions, orientations and optical parameters of the camera and projectors are unknown; the camera and projector optics can be modeled by perspective transforms; and that the projection screen is flat. Therefore, the various transforms between camera, screen and projectors can all be modeled as 2-D planar homographies:

$$
\left(\begin{array}{c}
x w \\
y w \\
w
\end{array}\right)=\left(\begin{array}{lll}
p_{1} & p_{2} & p_{3} \\
p_{4} & p_{5} & p_{6} \\
p_{7} & p_{8} & p_{9}
\end{array}\right)\left(\begin{array}{c}
X \\
Y \\
1
\end{array}\right),
$$

where $(x, y)$ and $(X, Y)$ are corresponding points in the camera and projector frames of reference, and $\vec{p}=\left(p_{1} \ldots p_{9}\right)^{T}$, constrained by $|\vec{p}|=1$, are the parameters specifying the homography. These parameters can be obtained from as few as four point correspondences, using well known cameraprojector calibration techniques [16,17].

One method to determine the homography for each camera-projector pair $T_{c, P_{i}}$ is to project a rectangle from the projector into the environment. The coordinates of the rectangle's corners in projector coordinates $\left(x_{i}, y_{i}\right)$ are known a priori, and the coordinates of the corners in the camera frame $\left(X_{i}, Y_{i}\right)$ are located using standard image processing techniques. ${ }^{2}$

The user can interactively specify the display area by manipulating the outline of a projected quadrilateral until it appears as a rectangle of the desired size and position on the display surface. This directly specifies the homography between the selected projector and the screen $T_{P_{i}, s}$; the outline of the selected rectangle can then be detected in the camera image as discussed above to determine the camera to screen homography $T_{c, s}$.

The projector-screen homographies $T_{P_{i}, s}$ model the geometric distortion (keystone warping) that is induced when an image is projected from an offcenter projector $P_{i}$. This distortion can be corrected by projecting a pre-warped image, generated by applying the inverse transform $T_{P_{i}, s}^{-1}$ to the original image. $^{3}$

Since $T_{P_{i}, s}^{-1} T_{P_{i}, s}=I$, one can see that the prewarping also aligns the images from different projectors so that all are precisely projected onto $S$. Applying the homographies derived from camera images, a multi-projector array can thus be efficiently configured to eliminate keystoning distortions and redundantly illuminate the display surface. In practice, our system is able to achieve alignment within one pixel.

\section{Active Virtual Rear Projection - SHADOW ELIMINATION}

By adding a camera or other sensor (Figure 3) that is able to detect the shadows on the display surface it is possible to dynamically correct penumbral shadows by projecting additional light into the

\footnotetext{
${ }^{2}$ Hough-transform line-fitting [18] locates the edges of the quadrilateral, and its corner coordinates are given by intersecting these lines.

${ }^{3}$ In our system, this pre-warp is efficiently implemented using the texture-mapping operations available in standard 3-D graphics hardware.
} 

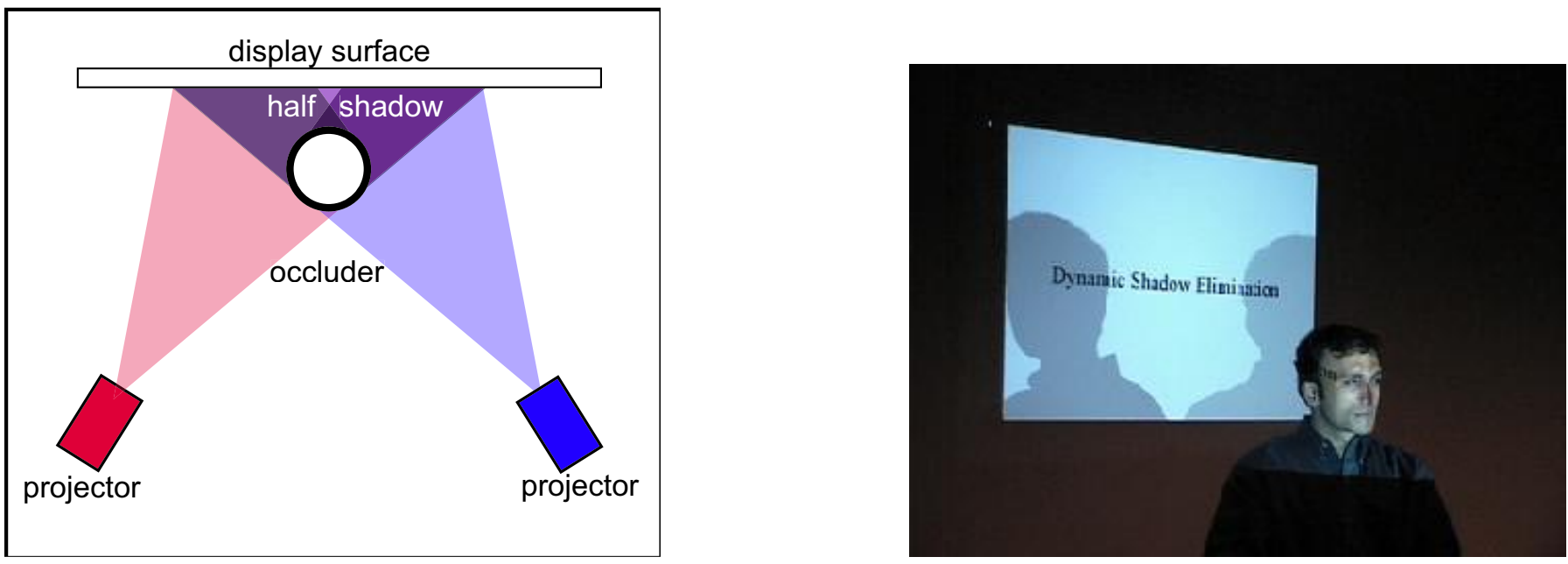

Fig. 2. Left: Passive Virtual Rear Projection. Right: Graphics within the penumbral shadows are still clearly visible although the user is subject to blinding light.

region from one of the non-occluded projectors. This Active Virtual Rear Projection (AVRP) system must precisely adjust projector output to compensate for each occlusion. If too little light is added, the shadow will remain visible; if too much light is used, over-illumination artifacts will be created. The shadow boundaries must be treated carefully since humans are very sensitive to edge artifacts.

\section{A. Occlusion detection}

The AVRP system focuses exclusively on detecting artifacts on the display surface. These can occur for either of two reasons. First, uncorrected penumbral occlusions appear as darker regions in a camera image that can be corrected by projecting additional light into the region. Second, artifacts may be caused by over-illumination of the display area, and occur most often when an occluding object (whose shadows had been eliminated) moves away suddenly. These bright spots are corrected by reducing the light intensity in the region. Our shadow elimination algorithm makes no assumptions about the locations, sizes or shapes of occluders.

Figure 4 illustrates the algorithm. During its initialization phase (when the scene is occluderfree) the system projects each image it wishes to display and captures several camera images of the projected display. These images are pixel-wise averaged to create a reference image for that slide, and this image represents the desired state of the display (Figure 4, top left). The goal of occlusion detection is to identify regions in the current image that deviate from this ideal state. During operation, the system camera acquires a current image of the projected display which may contain uncorrected shadows. For example, the image shown in Figure 4 (center left) has two dark regions, corresponding to the two penumbrae cast by one person standing in front of the display (each projector creates one shadow).

Since the display surface remains static, a pixelwise image difference between current and reference camera images can be used to locate shadows and over-compensation artifacts. To reduce the effects of camera noise and minor calibration errors, we apply a $5 \times 5$ spatial median filter to the difference image. A negative value in a difference image pixel means that the corresponding patch on the screen was under-illuminated in the current image. This information is represented in terms of an alpha mask $\left(\alpha_{t}\right)$, which when applied to the current camera image, should bring it closer to the reference image. Alpha values range from 0 (dark) to 255 (bright), and the mask is initialized to 128 at $t=0$. The alpha mask is updated at every time-step using the following simple feedback system:

$$
\alpha_{t}(x, y)=\alpha_{t-1}(x, y)-\gamma\left(I_{t}(x, y)-I_{0}(x, y)\right),
$$

where $I_{t}$ is the camera image at time $t, I_{0}$ is the reference image, and $\gamma$ is a system parameter (set to 0.3 in our implementation). For a static scene, the alpha mask converges to a stable fixed point in a very short period of time.

A noteworthy point about our shadow elimination system is that all of the projectors in the 

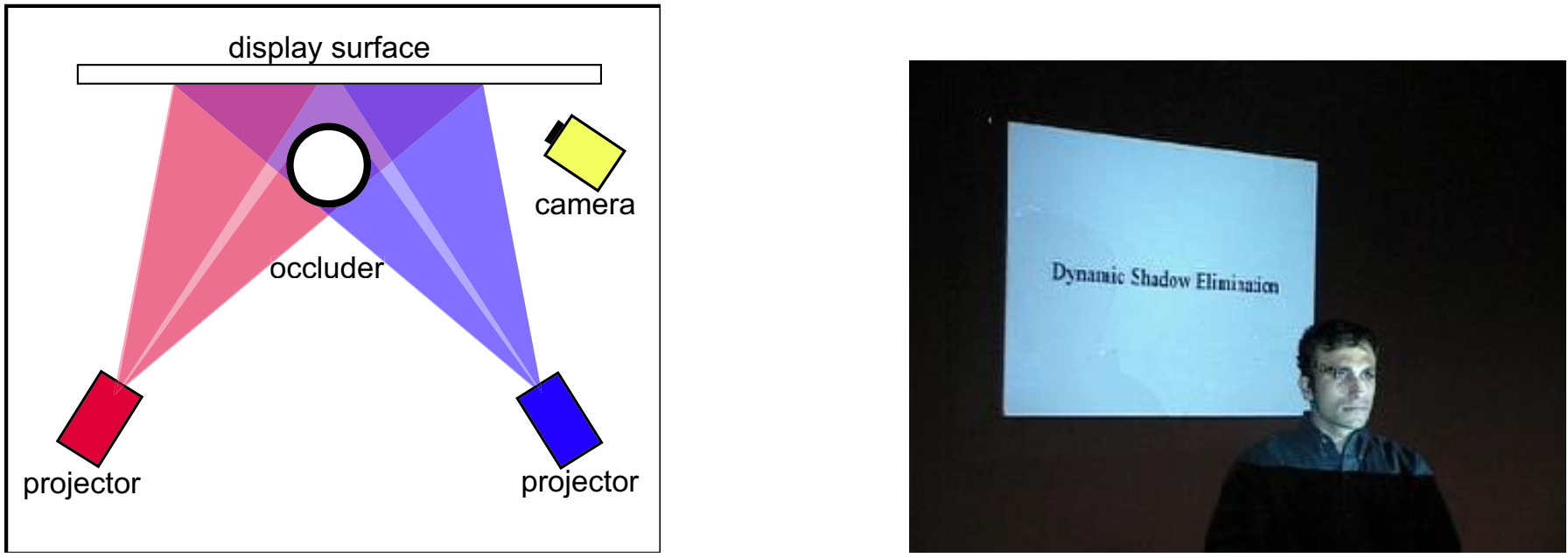

Fig. 3. Left: Active Virtual Rear Projection (AVRP). Right: Penumbral shadows are eliminated but the blinding light remains.

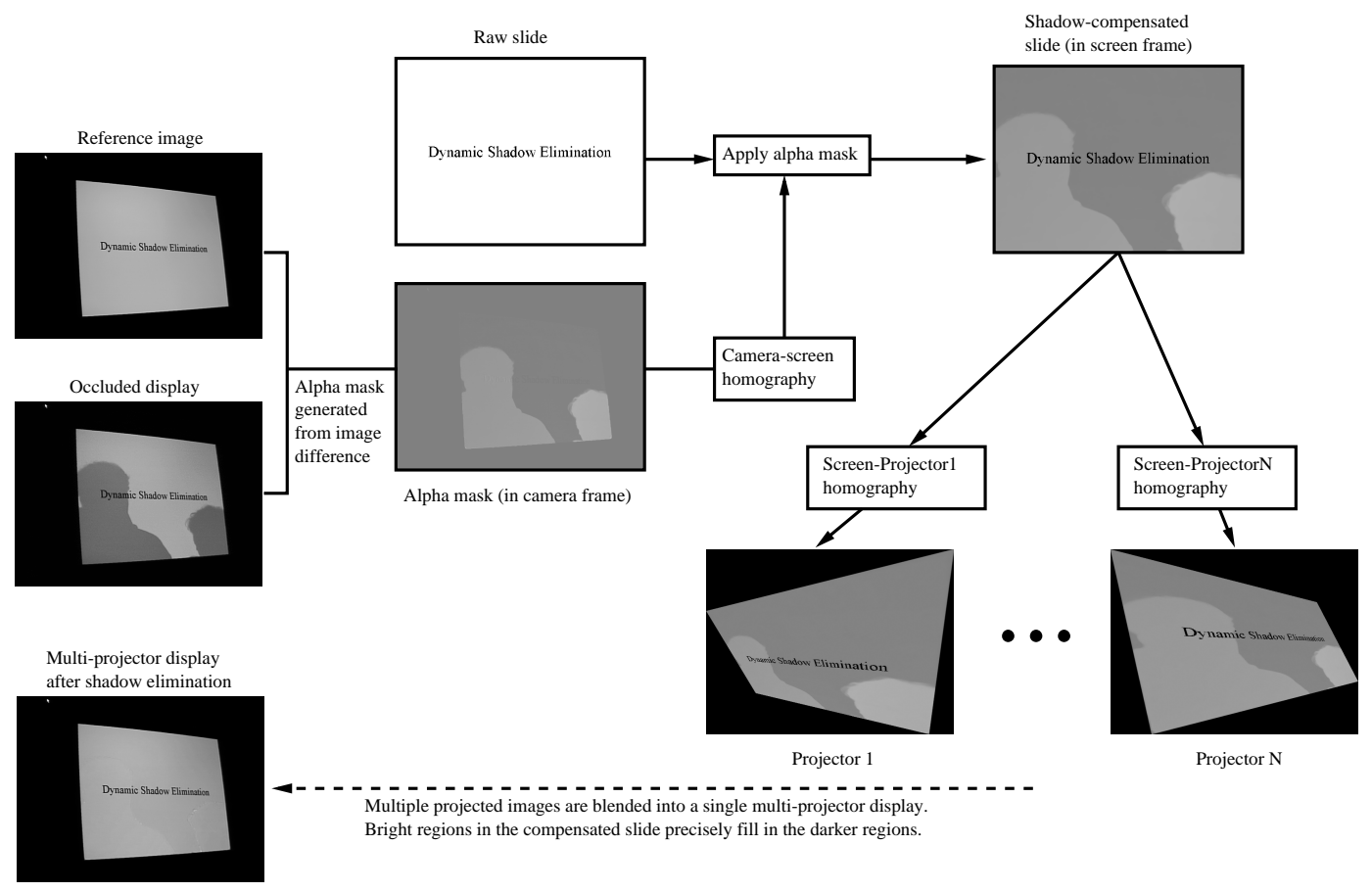

Fig. 4. This diagram summarizes the occlusion detection and shadow elimination algorithms. The images in the left column were taken by the system camera during operation. The two penumbral occlusions caused by the person blocking both projectors are identified and corrected to create a shadow-free display (bottom left). See text for details.

multi-projector system use the same alpha mask for shadow removal. This reduces the amount of processing required, but results in additional light being projected onto occluders as described below.

\section{B. Shadow elimination}

The alpha mask (described above) integrates the previous state of the shadow correction, and information from the current difference image. However, since it was computed in the camera frame of reference, it must be transformed into the screen frame of reference before it can be applied; this is done using the camera-screen homography $T_{c, s}$ discussed in Section III-A.

It is surprising that using the same alpha mask for all projectors correctly eliminates all of the penumbral shadows! This can be explained by the following argument. Consider the two-penumbra shadow configuration generated by the two-projector, oneoccluder system shown in Figures 2 (right) and 4. From P1's perspective, the left high-alpha region 
falls precisely on the left penumbra (Shadow2) while the right high-alpha region simply overilluminates the occluder. From P2's perspective, the left high-alpha region falls on the occluder (without effect) and the right one corrects for the right penumbra (Shadow1). Thus, both projectors are able to use the same image to eliminate shadows.

Since this algorithm does not use photometric models of the environment, projectors or camera, it cannot predict precisely how much light is needed to remove a shadow. However, the iterative feedback loop used to update the alpha mask allows us to avoid this problem: the system will continue adding light to shadowed regions until the region appears as it did in the reference image. This approach has additional benefits. For instance, the system is able to correct for the fuzzy occlusions caused by area light sources (e.g., the diffuse shadow created by a hand moving near the projector) without requiring an explicit model of the shadow formation process. One drawback to such an iterative technique is that the alpha mask can require several iterations to converge; in practice, shadows are eliminated in approximately 3 iterations. The second drawback of this form of active virtual rear projection with shadow elimination is that it indiscriminately projects additional light onto the occluder (user) as well as the areas of shadow on the display surface. If the user turns to face the projectors this blinding light is distracting [19].

\section{Blinding Light SUPPRESSION}

To combat this blinding light being cast upon users, we must be able to determine which pixels in each projector are falling upon occluders. After the projectors have been geometrically aligned, we can easily determine which source pixels from the projectors contribute to the intensity of an arbitrary screen pixel. In the following analysis, we assume that the contributions are at some level additive. Given $N$ projectors, the observed intensity $Z_{t}$ of a particular screen pixel at time $t$ may be expressed by

$$
Z_{t}=C\left(k_{1 t} S_{1}\left(I_{1 t}\right)+\cdots+k_{N t} S_{N}\left(I_{N t}\right)+A\right),
$$

where $I_{j t}$ is the corresponding source pixel intensity set in projector $j$ at time $t, S_{j}(\cdot)$ is the projector to screen intensity transfer function, $A$ is the ambient light contribution, assumed to be time invariant,
$C(\cdot)$ is the screen to camera intensity transfer function and $k_{j t}$ is the visibility ratio of the source pixel in projector $j$ at time $t$. Note that all the variables and functions also depend on the spatial position of the screen pixel, but this is omitted from the notation since we will consider each pixel in isolation. See figure 5.

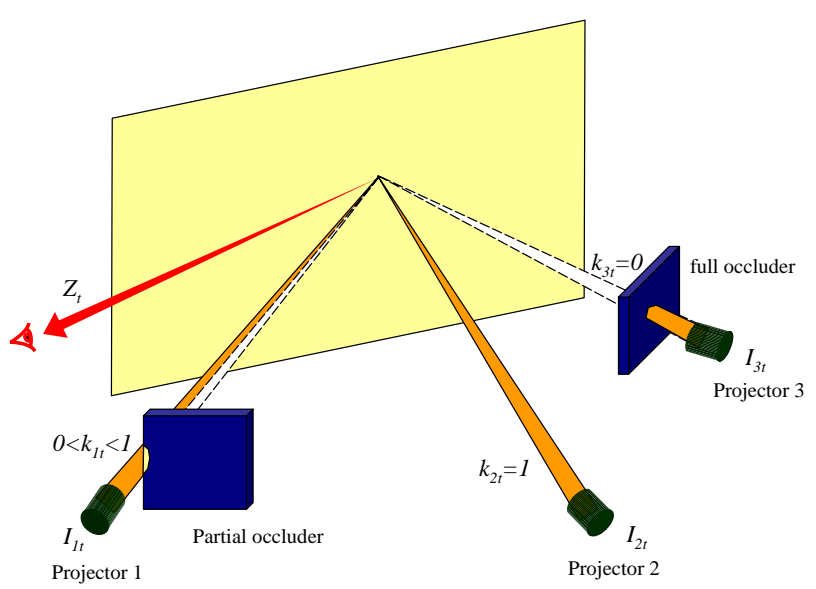

Fig. 5. Photometric framework. This diagram illustrates equation (2), in which the observed display intensity $Z_{t}$ is related to the combination of projector source pixels $I_{j t}$ and the corresponding visibility ratios $k_{j t}$. The visibility ratios vary accordingly with nonocclusion, partial and full occlusion.

When occluders obstruct the paths of the light rays from some of the projectors to the screen, $Z_{t}$ diminishes and shadows occur. This situation is quantitatively modeled via the visibility ratios, which represent the proportion of light rays from corresponding source pixels in the projectors that remain unobstructed. If the projectors were modeled as point-light sources, occluders would block either none or all of the light falling on a given pixel from any particular projector; therefore, $k_{j t}$ would be a binary variable. However, this assumption is not valid in real-world conditions. Our system must cope with partial occluders (created by objects near the projector) that cast fuzzy-edged shadows on the screen. In these cases $k_{j t}$ denotes the degree of occlusion of projector $j$ for the given pixel.

\section{A. Occlusion detection}

The AVRP-BLS system focuses exclusively on detecting deviation of the observed intensities on the screen from the desired intensities when occluders are not present. The major cause of deviation is occlusion, although deviation can also occur because of changes in ambient lighting, projector failure, 

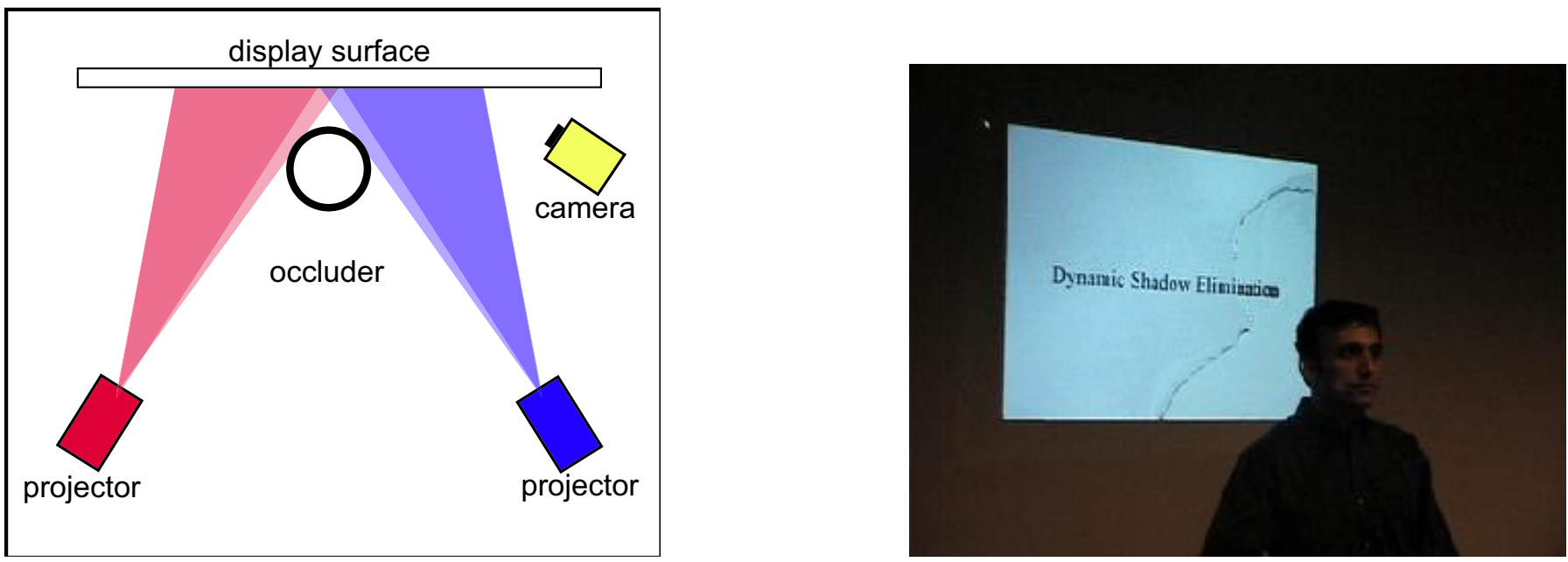

Fig. 6. Left: AVRP with Blinding Light Suppression. Right: Light is kept off of occluders face.

etc. Our system can handle all of these problems (as discussed in the next section). No assumptions are made about the locations, sizes or shapes of occluders.

Mathematically, the desired intensity of a particular screen pixel may be represented by $Z_{0}$. This may be obtained in the initialization phase when the system projects each presentation slide and captures several camera images of the projected display while occluders are absent. As an occluder is introduced in front of projector $k$ to create penumbral shadows, the visibility ratio $k_{j t}$ decreases, such that $k_{j t}<1$. Hence $Z_{t}<Z_{0}$. These deviations in the screen can be detected via a pixel-wise image difference between current and reference camera images to locate shadow artifacts.

\section{B. Iterative Photometric Compensation}

Our system handles occluders by

1) compensating for shadows on the screen by boosting the intensities of un-occluded source pixels; and

2) removing projector light falling on the occluder by blanking the intensities of occluded source pixels.

The degrees-of-freedom available to us are the source pixel intensities $I_{j t}$, which may be changed. Hence for a shadowed screen pixel where $Z_{t}<Z_{0}$, we ideally want to compensate for the shadow (i.e. setting $Z_{t+1}=Z_{0}$ ) by (i) increasing $I_{j(t+1)}$ to be larger than $I_{j t}$ if $k_{j t}=1$, and (ii) reducing $I_{j(t+1)}$ to zero if $k_{j t}<1$.
However, it is very difficult to accurately model $C(\cdot)$ and $S_{j}(\cdot)$. Even if we know the exact values for the ambient lighting and visibility ratios, it is almost impossible to update the source pixels such that in one time step the shadows are eliminated. Fortunately, we expect $C(\cdot)$ and $S_{j}(\cdot)$ to be positive monotonic, and an iterative negative feedback loop can be used to compute $I_{1 t}, \ldots, I_{N t}$ required to minimize $Z_{t}-Z_{0}$.

The advantages of such a system are:

- it does not require explicit modeling of $C(\cdot)$ and $S_{j}(\cdot)$,

- it does not require explicit measurement of the visibility ratios $k_{j t}$,

- it is able to handle slowly varying ambient light.

As in Section IV, the change in the intensity of each source pixel in each projector is controlled by the alpha value associated with the pixel:

$$
I_{j t}=\alpha_{j t} I_{0}
$$

where $I_{0}$ is the original value of the source pixel (i.e. pixel value in the presentation slide) and is the same across all projectors, while $\alpha_{j t}$, which can vary between 0 and 1 , is the time-varying, projector-dependent alpha value. The alpha values for the source pixels in one projector are collectively termed the alpha mask for the projector.

The earlier AVRP system described in Section IV can compensate for shadows but is incapable of suppressing projected light falling on the occluder. In particular, that simpler method cannot distinguish between the contributions of individual projectors. 
Instead, all projectors boost their pixel intensities for each occluded region. This has two undesirable consequences: (1) bright "halos" may appear around eliminated shadows, particularly when occluders are in motion; and (2) the amount of distracting light projected on users is increased rather than reduced by the system. This motivates the need for a more complex solution where the alpha masks are different for different projectors.

The approach adopted here is to design components which separately handle the problems of shadow elimination and occluder light suppression, and integrate them into a complete system. These are discussed in the following sections.

\section{Shadow Elimination}

Eliminating shadows involves increasing values for corresponding source pixels. The shadow elimination (SE) component of the system is based on

$$
\left(\Delta \alpha_{j t}\right)_{\mathrm{SE}}=-\gamma\left(Z_{t}-Z_{0}\right)
$$

where $\Delta \alpha_{j t}=\alpha_{j(t+1)}-\alpha_{j t}$ is change of $\alpha_{j t}$ in the next time-frame, and $\gamma$ is a proportional constant $(\gamma$ is 0.7 in our implementation). This component is a simple, linear feedback system.

\section{Blinding Light Suppression}

Suppressing projector light falling on occluders involves diminishing the source pixels corresponding to the occluded light rays. We determine whether a source pixel is occluded by determining if any changes in the source pixel result in changes in the screen pixel. However, since there are $N$ possible changes of source pixel intensities from $N$ projectors but only one observable screen intensity, we need to probe by varying the source pixels in different projectors separately. This cyclical probing results in a serial variation of the projector intensities.

The light suppression (LS) component of the system is based on

$$
\left(\Delta \alpha_{j t}\right)_{\mathrm{LS}}=-\beta \frac{\Delta \alpha_{j(t-N)}^{2}}{\Delta Z_{t}^{2}+\epsilon},
$$

where $\Delta Z_{t}=Z_{t}-Z_{t-N}$ is the change in the screen pixel intensity caused by the change of alpha value $\Delta \alpha_{j(t-N)}$ in the previous time frame when projector $j$ is active, $\beta$ is a small proportional constant and $\epsilon$ is a small positive constant to prevent a null denominator ( $\beta$ and $\epsilon$ are 0.1 in our implementation).

The rationale for (5) is that if the change in $\alpha_{j t}$ results in a corresponding-sized change in $Z_{t}$, the subsequent change in $\alpha_{j t}$ will be relatively minor (based on a small $\beta$ ). However if a change in $\alpha_{j t}$ does not result in a change in $Z_{t}$, this implies that the source pixel is occluded. The denominator of (5) approaches zero and $\alpha_{j t}$ is strongly reduced in the next time frame. Hence occluded source pixels are forced to black.

Note that the probe technique must be employed during shadow elimination as well. In particular, the system must be able to discover when a pixel which was turned off due to the presence of an occluder is available again, due to the occluders disappearance. This constraint is smoothly incorporated into our algorithm.

\section{E. Integrated System for Shadow Elimination and Blinding Light Suppression}

The integrated iterative feedback system combines (4) and (5) to get

$$
\Delta \alpha_{j t}=\left(\Delta \alpha_{j t}\right)_{\mathrm{SE}}+\left(\Delta \alpha_{j t}\right)_{\mathrm{LS}} .
$$

The alpha values are updated within limits such that

$$
\alpha_{j t}=\left\{\begin{array}{cl}
1, & \text { if } \alpha_{j t}+\Delta \alpha_{j t}>1, \\
0, & \text { if } \alpha_{j t}+\Delta \alpha_{j t}<0, \\
\alpha_{j t}+\Delta \alpha_{j t}, & \text { otherwise. }
\end{array}\right.
$$

The following synthetic example illustrates the system. For a particular screen pixel at a typical steady state when shadows have been eliminated, suppose the corresponding source pixel at projector 1 has $\alpha_{1 t}=0$ and the source pixel at projector 2 has $\alpha_{2 t}=1$. At this state, $Z_{t}=Z_{0}$ and $\Delta \alpha_{j t}=0$ via (6). If source pixel 2 is suddenly occluded and source pixel 1 is un-occluded, then $Z_{t}<Z_{0}$ because source pixel 1 is still black. However, $\Delta \alpha_{1 t}$ becomes dominated by $\left(\Delta \alpha_{1 t}\right)$ SE which forces source pixel 1 to be bright. On the other hand, $\Delta \alpha_{2 t}$ becomes dominated by $\left(\Delta \alpha_{2 t}\right)_{\mathrm{LS}}$ since the screen pixel does not change when $\alpha_{j t}$ is changed. This forces source pixel 2 to be dark. Note that even when source pixel 2 becomes un-occluded, nothing changes if source pixel 1 remains un-occluded since the shadows have already been satisfactorily eliminated. See figure 7 . This particularly illustrates the hysteresis effect in which source pixels are not boosted or blanked until 
new shadows are created - the system does not automatically return to an original state, nor change as a result of deocclusion.

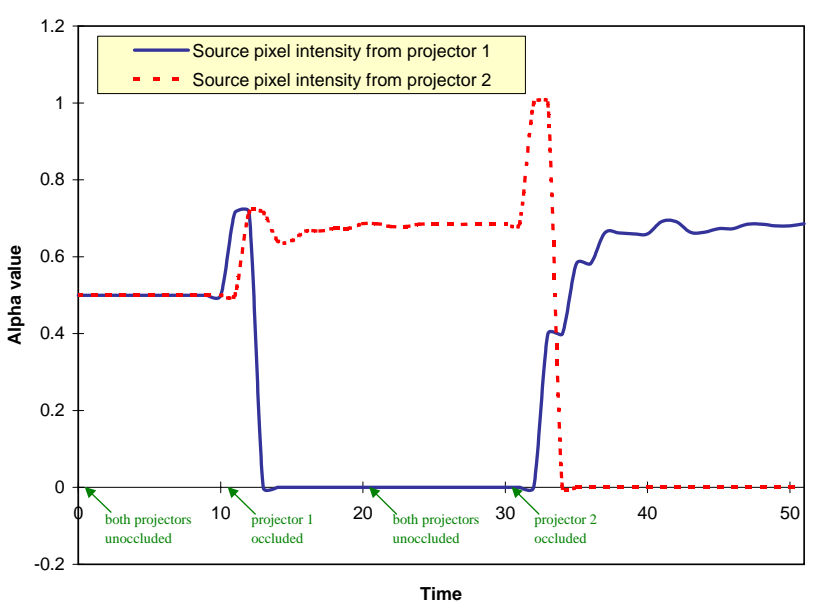

Fig. 7. Synthetic example of transitions in projector source pixel intensities. This graph shows the intensity transition of two corresponding projector source pixels over time, subject to four events of occlusions and deocclusions. Note the hysteresis effect in which the source pixels are not boosted or blanked until new occlusion events occur.

Since we do not have good photometric models of the environment, projectors or camera, we cannot predict precisely how much light is needed to remove a shadow. However, the iterative feedback loop used to update the alpha mask allows us to avoid this problem: the system will continue adding light to shadowed regions until the region appears as it did in the reference image. Similarly, the system will blank projector source pixels which are occluded and do not affect the observed images. This approach has additional benefits. For instance, the system does not require an accurate photometric model of the shadow formation process to correct for occlusions with non-binary visibility ratios, e.g. the diffuse shadow created by a hand moving near the projector. The drawback to such an iterative technique is that the alpha mask can require several iterations to converge; in practice, shadows are eliminated in approximately 5-7 iterations.

In our software only implementation, the AVRPBLS system is able to calculate 1.6 iterations per second (See Table I.) Even assuming advances in processing power, when using commodity projectors, which are limited to 60 or $85 \mathrm{fps}$, a series of 5-7 iterations would produce a visual artifact for up to
$1 / 10^{\text {th }}$ of a second. ${ }^{4}$ There are two possible solutions to making the changes to the display unnoticeable to humans. The first method is to greatly increase the speed of the entire feedback loop. This would require projectors and cameras which operate at $120 \mathrm{fps}$ or faster. The second method is to detect the occluder instead of the occlusion (shadow) and use that knowledge to correct the occlusion as (or before) it occurs.

\section{SWITCHING VRP}

The previous systems provide redundant illumination to each pixel from multiple projectors, dynamically adjusting the amount of illumination from each projector on a per-pixel basis based upon the feedback provided by a camera observing the projected display.

The downside of these approaches is that they assume that the camera has an un-occluded view of the display surface. We can relax this assumption by detecting the occluder instead of the occlusion (shadow). However, as we would no longer have an un-obstructed view of the display, we will have to correct the projector's output blindly, without feedback. To do this successfully, each pixel on the display surface is illuminated by only one projector at a time. As the projector illuminating a pixel is occluded, responsibility for illuminating that pixel is shifted to another (un-occluded) projector. This presents several challenges:

1) The system must know which pixels are occluded for at least $N-1$ of the $N$ projectors in the system, so that it can correctly assign pixel regions to unoccluded projectors to ensure that a complete image appears on the display surface regardless of occlusions which may partially block portions of each projector.

2) The output from all projectors must be photometrically uniform, so that any projector can "fill in" for any other projector without a noticeable change in intensity or color.

3) The sub-images projected from each projector must overlap in such a way as to produce a uniform output image without visible seams or intensity/color shifts. To achieve this, the edges of each image must be blurred so that they blend together imperceptibly.

\footnotetext{
${ }^{4}$ As with AVRP, the largest intensity changes happen in the first or second iteration. As the iterative feedback loop converges, subsequent iterations are much less noticeable.
} 

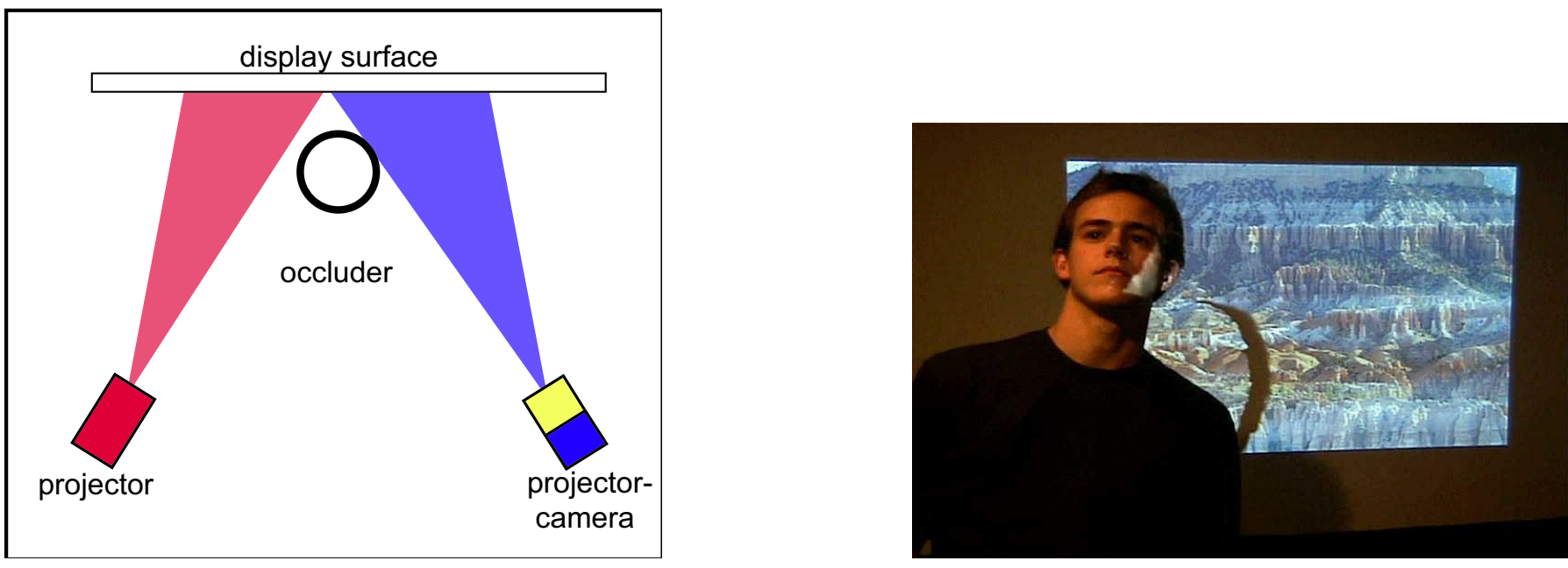

Fig. 8. Left: Switching VRP. Right: Shadows are eliminated and blinding light is suppressed with a moving user. The gap in the display caused as the user moves into the scene will be corrected in the next iteration.

\section{A. Occlusion Detection}

In our approach, we position a camera close to the projector lens of the primary projector so that detected occluder silhouettes align with corresponding projector mask silhouettes with little to no parallax effects caused by projector-camera disparity. If the optical axes of the projector and camera are aligned by means of a beam-splitter, parallax effects are eliminated [20]. To simplify the detection of occluders, the camera is filtered to detect only infrared light and the display surface is illuminated with infrared lights. Background subtraction of the IR camera images is not affected by light projected from the projectors and, as shown in Figure 11(b), the back-lit silhouette of occluders creates a strong contrast between foreground and background.

Because we are detecting occluders (instead of shadows) we do not need to pre-shoot background plates for each expected frame [21] or predict the expected appearance of each image when projected onto the display surface [22].

For each compensation step, the IR camera image must be processed to meet the challenge of preserving high image quality in the face of varying pixel-projector ownership. These steps are illustrated in Figure 11. First, the acquired image must be warped to align with the display surface using a camera-surface homography. Second, the image is segmented into occluder and non-occluder regions. Our implementation uses background subtraction. In some cases, median filtering is needed for noise removal, but in our experiments the back-lit oc- cluders were easily segmented without noise. Third, the occluder regions are dilated to allow a region of tolerance for occluder movement between each compensation step. Finally, the mask is blurred to blend seams between projectors. Figure 9 illustrates the necessity for blending to avoid distracting seams.
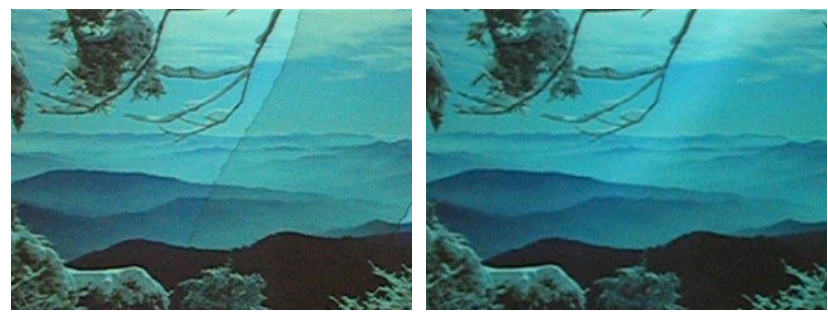

Fig. 9. Boundary between regions of varying projector ownership. Left: before seam blending Right: after seam blending

\section{B. Photometric Uniformity}

The projected display from one projector must appear photometrically uniform to another projector to insure the VRP displays consistently. Calibration for photometric uniformity is necessary to make the hand-off of a pixel from one projector to another unnoticeable.

Majumder and Stevens have found that the major source of apparent color variation across multiple projectors is primarily due to luminance variation, and that the chrominance of projectors (of the same brand) are very similar [23,24]. Their work has focused on tiled multi-projector displays where 
the projectors are oriented perpendicular with the display surface.

In a virtual rear projection system, the projectors are oriented as much as $50^{\circ}$ from the normal, with a $30^{\circ}$ to $45^{\circ}$ off-axis orientation being typical. This extreme angle causes drastic changes in the level of illumination from each projector across the display surface. The side of the display surface closer to the projector is over-illuminated, while the far side is under-illuminated. This angle-induced ramping function is in addition to the variations in projector illumination found by Majumder and Stevens.

To correct for the intensity variance in our VRP system, we use luminance attenuation (alpha) masks which modify the intensity of each projector pixel so that all pixels are evenly illuminated, regardless of their location on the display surface or which projector is currently being used to illuminate the pixel.

The method we use to generate the attenuation maps is similar to those used by Majumder and Stevens for their Luminance Attenuation Maps (LAM) [25] except that it does not require a calibrated projector or camera. The darkest intensity measured when projecting white from each projector independently is set as a target. All pixels are iteratively reduced in intensity one step at a time (to account for non-linear projector and camera responses) until the target intensity is uniform across the display. Figure 10 shows two example LAMs and the following pseudocode describes our simple algorithm for their creation:

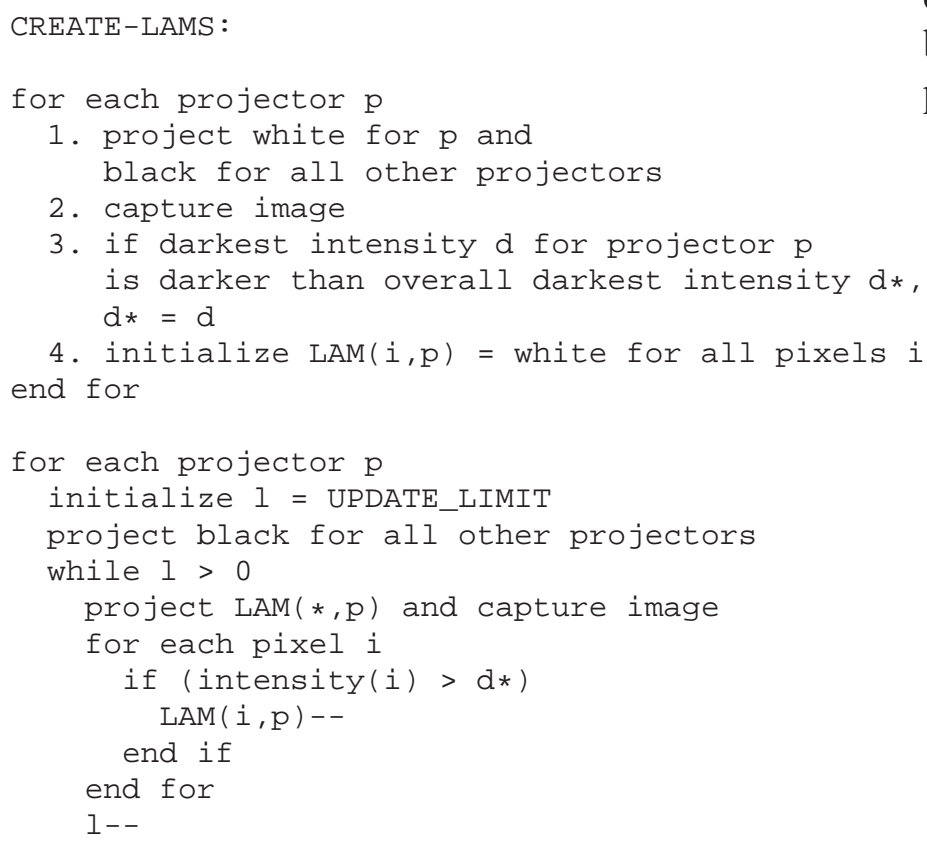

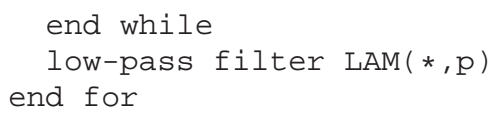

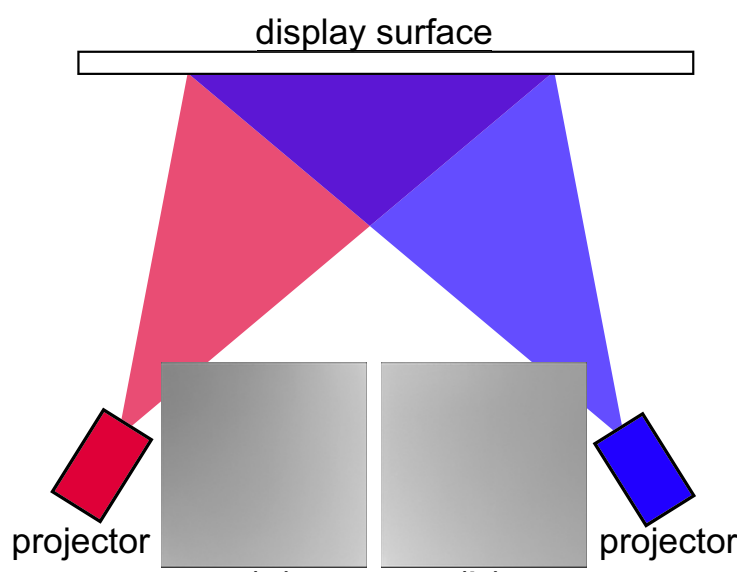

(a)

(b)

Fig. 10. Luminance Attenuation Maps (LAMs): (a) LAM for projector positioned to left of projection surface (b) LAM for projector positioned to the right of the projection surface. Note that the dark regions of each LAM correspond with the shortest projection distance to the display surface.

\section{Edge Blending}

We assume that the output image from each projector is already geometrically aligned on the display surface and the output of each projector has been modified to be as photometrically uniform as possible. Our goal is to project portions of the image from different projectors while retaining a final displayed image that appears uniform and without edge artifacts. This can be achieved by using edge blended alpha masks to limit the output of each projector, generated as follows:

1) Order your projectors from $P_{0} \ldots P_{N}$. Projector $P_{0}$ will be initially responsible for the whole display. As it is occluded, projector $P_{1}$ will be used to fill-in occluded regions. Any regions occluded in both projector $P_{0}$ and $P_{1}$ will be handled by projector $P_{2}$ and so on. Associate an initially zero alpha mask with each projector $\alpha_{0} . . \alpha_{N}$ which will be used to control the active output pixels.

2) Generate an occlusion mask $O_{0} . . O_{N}$ for each projector, indicating which projector pixels are occluded.

3) For the alpha mask of the ith projector $\alpha_{0<i<=N}$ turn on all pixels which are not occluded in the occlusion mask $O_{i}$ and have 
not already been turned on in any previous alpha masks $\alpha_{0 . . i-1}$. This results in a set of mutually exclusive alpha masks which favor projectors based on their ordering. A pixel must be occluded in all projectors before it will be lost.

4) We then perform the following operations on each alpha mask to add a feathered edge which hides the seam.

- Filter each alpha mask $\alpha_{0} . . \alpha_{N}$ with a $3 \times 3$ median filter to remove noise.

- Dilate each alpha mask three times to expand their extent.

- Blur the expanded alpha masks with a Gaussian filter to feather their edges.

When the occluders are correctly detected, the result of using these alpha masks to control the output of the projectors is a projected display that appears seamless and shadow free.

\section{Improving Performance Using the GPU}

As users move in front of an active VRP display, they may cast new shadows by moving faster than the system can update the screen. This occurs when the users move outside of the region of tolerance created by the dilation operation before the display is updated. Increasing the system frame-rate and decreasing system latency enables users to make quick natural movements such as emphasizing a point with a fast hand gesture. The image processing steps needed for switched VRP may be optimized by exploiting today's programmable graphics cards (GPUs). Image processing on the GPU shifts the speed limit of switched VRP away from computation on the CPU to capture and display rates of the camera and projector. Figure 11 illustrates our image processing pipeline using the GPU.

There are three capabilities of GPUs and DirectX 9.0 that we exercise in order to eliminate the bottleneck of image processing: (a) multiple render targets, (b) pixel shaders and (c) multi-head resource sharing. First, the Multiple Render Targets (MRT) capability provided with Direct3D version 9.0 enables us to store the results of each image processing step in an off-screen rendering surface for succeeding filter operations to use as input. By setting the texture coordinates $(\mathrm{u}, \mathrm{v})$ of a screenaligned quadrilateral to correspond with the camera image coordinates $(\mathrm{x}, \mathrm{y})$ of the projected display, the camera-surface warp may be performed by rendering the quadrilateral texture-mapped with the camera image. The warped texture is now available on an off-screen surface for subsequent filtering using pixel shaders.

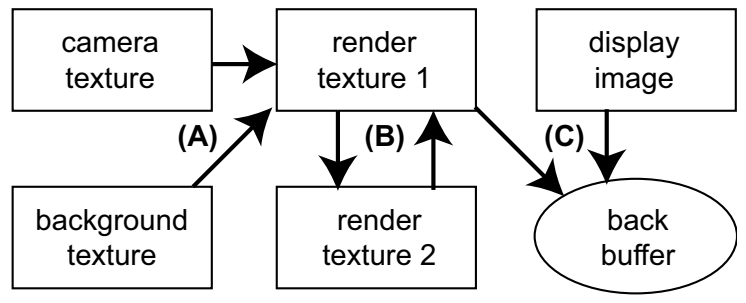

Fig. 12. Pixel Shader Pipeline: Boxes represent textures and arrows denote texture sampling operations used in pixel shaders. (a) Background subtraction shader stores result in render texture 1. (b) Render textures 1 and 2 are used as sampling buffers for dilation and blurring operations, each of which require 2 independent shaders (c) The final occluder mask is composited with a display texture and rendered into the DirectX back buffer for display.

The second capability provided by GPUs is fast image processing using pixel shaders. Background subtraction, dilation, median filtering and blurring may be implemented as pixel shader programs [26]. These pixel shaders were written in DirectX HighLevel Shader Language (HLSL). Using two texture samples and a threshold, the result of a background subtraction shader is stored in the first of two offscreen render targets. Next, dilation is performed using two separate pixels shaders. The first shader dilates the result of background subtraction using 1D texture samples horizontally and the second dilates the resulting texture vertically. Separating dilation into two operations decreases the number of required texture samples and improves performance from $O\left(n^{2}\right)$ to $O(n)$. To further improve processing time, the two off-screen render textures were reduced to a resolution of $128 \times 128$ pixels (to be subsampled during compositing operations). Following dilation, blurring is performed in a similar manner using two separate shaders. Finally, the resulting occluder mask is composited with the display frame using one pixel shader. The interaction between each pixel shader and the input / output textures used by them is illustrated in Figure 12.

Finally, multi-head resource sharing in DirectX 9 makes it possible to use one rendering device across multiple display heads. Previously, each head required its own device and therefore needed separate sets of textures and pixel shader computations. By using one device instead of two, some of the 


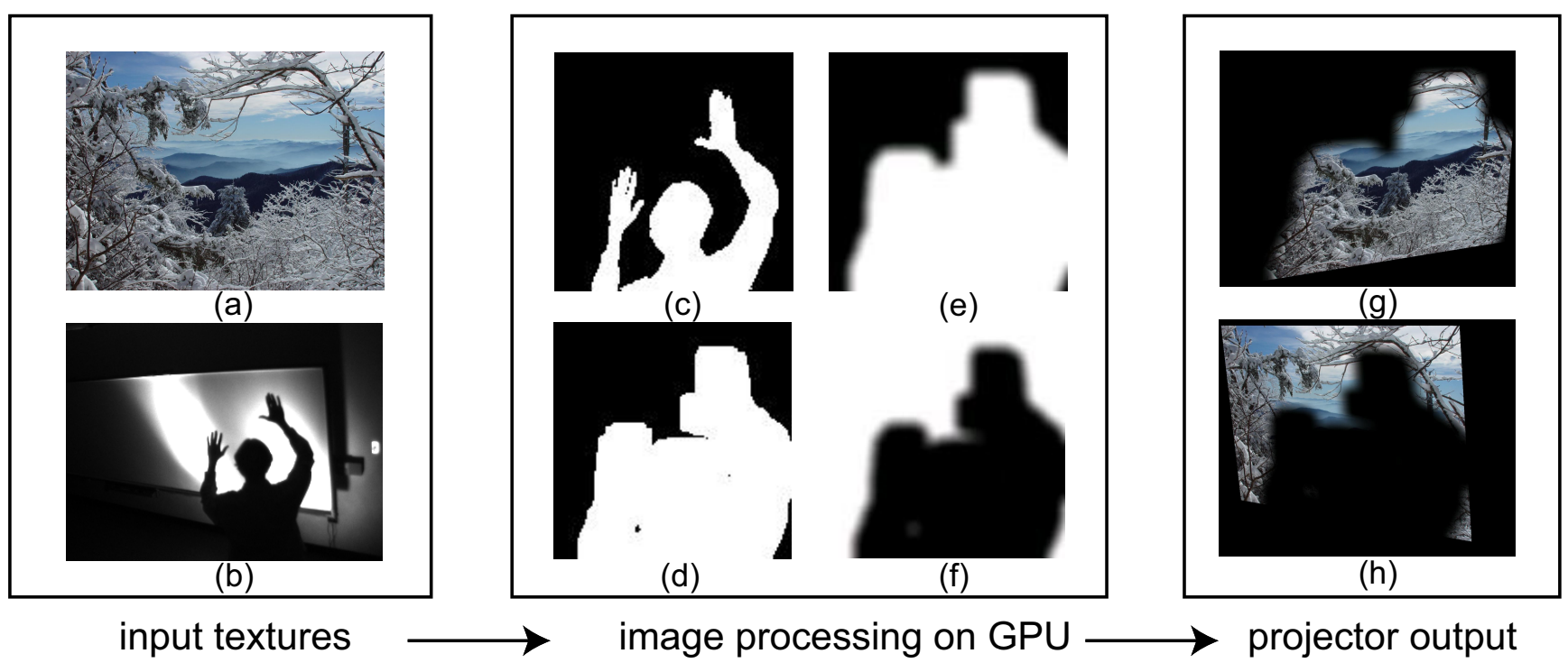

Fig. 11. GPU-centric architecture: (a) display texture (b) IR camera frame (c) occluder mask texture (d) dilated mask to tolerate inter-frame occluder movement (e) blurred mask for projector 1 blending (f) blurred mask for projector 2 blending (g) keystone-corrected projector 1 output (h) keystone-corrected projector 2 output

pixel shaders need only be executed once saving time and texture memory. A background subtraction and dilation pixel shader computation is removed. An initial dilation of $n$ pixels is performed to permit sufficient occluder movement within frame updates.

A second dilation of $k$ pixels is needed to overlap projector masks before blending. Before multi-head resource sharing, one display device performed $2 n$ texture samples and the other sampled $2(n+k)$ pixels $(4 n+2 k$ total samples). After multi-head sharing, a dilation using $2 n$ texture samples is shared among both display heads and a remaining $2 k$ pixels are sampled for the overlapping region $(2 n+2 k$ total samples), saving $2 n$ texture samples per pixel. Following dilation, blurring and compositing operations must be performed for each display head separately due to differences between the occluder masks.

\section{RELATED WORK}

The 3M Digital Wall Display and the NEC WT-600 projector are commercial products which implement warped front projection using special optics $[14,15]$, but do not allow the user to position the display arbitrarily. Many projectors include a keystone correction feature implemented by a physical "lens-shift" which allows the image to be warped to a limited degree. New advanced video processing chips (such as the Silicon Optix Realta, including eWARP-2 Geometry Processing) are being integrated into high end projectors emerging in the market which support off axis projection of up to $\pm 45^{\circ}$ (vertical) and $\pm 35^{\circ}$ (horizontal). Modern graphics cards by nVidia support the nvKeystone feature, which allows users to warp a projected display arbitrarily. None of these commercially available solutions deal with the calibration of multiple displays to provide redundant illumination.

Much work has been done with multiple projectors to build tiled display walls $[1,2,3,4]$ and Chen et al. have used homographies to align multiple tiled projectors in a large-format display wall [27] but did not overlap projectors to provide redundant illumination.

Tan and Pausch used IR back-lighting and an IR camera to detect a person and create a black "mask" over projected graphics which solves the blinding light suppression problem [28]. A commercial appliance sold by iMatte uses a similar technique of masking out projected graphics, but has a more sophisticated segmentation algorithm, allowing it to work with a front projected IR pattern, eliminating the need for back-lighting. Both of these systems suppress blinding light, but do not compensate for shadows occurring on the display. Indeed, they actually slightly increase the size of the shadows on the display as the masks they generate are slightly larger than the users. They are most useful for applications where the user is not trying to interact with the displayed image (e.g. while giving a presentation).

Our previous work as described in Sec- 
tions IV and VI eliminated shadows, but required the collection of a reference image with no occluders present and assumed an unoccluded view to the display [29]. Researchers at the University of Kentucky developed a photometric model which they use to generate a reference image of arbitrary graphics, predicting how it should appear when projected [22]. But their system was too slow for interactive use, retained the assumption of an unoccluded view to the display, and did not solve the blinding light problem. Jaynes et al. updated this work to improve the speed to approximately nine frames per second, by updating bounding regions instead of individual pixels [30]. Similar to AVRP or AVRP-BLS, their system requires numerous frames to converge to a stable display. Their updated system still requires that cameras have an un-occluded view of the screen, and does not eliminate blinding light.

Our work as described in Section V solves the blinding light problem but was also slow and relied on an unoccluded view of the display. The switching system presented in section VI removes the unoccluded camera view requirement, and is currently limited by the projector frame-rate to 85 frames per second.

Majumder and Stevens used luminance attenuation maps in multi-projector tiled displays on a per-channel basis to correct for color nonuniformity [25,31], but their method for generating the LAM's required a full photometric model of the camera and projector(s) which required significant manual processing.

\section{EVALUATION}

To evaluate their relative performance, we performed an empirical evaluation of each of the algorithms discussed previously. In this experiment, each algorithm was run on the same hardware setup. After the algorithms had initialized, we collected a reference frame consisting of the average pixel values on the display with no occluders, and then paused the algorithm. We then introduced an occluder into the beam path of one projector and restarted the algorithm.

We used a static occluder which appeared (to the algorithms) instantaneously so that each algorithm would be measured under identical conditions. Because the tests can not be performed in a simulated environment, we were unable to physically replicate
TABLE I

Algorithm Performance Measures

\begin{tabular}{|c|c|c|c|c|c|}
\hline Condition & $\begin{array}{l}\text { Frames to } \\
\text { Converge }\end{array}$ & $\begin{array}{l}\text { SSD } \\
\text { Error }\end{array}$ & $\begin{array}{l}\text { Occluder } \\
\text { Light }\end{array}$ & F.P.S. & $\begin{array}{l}\text { Time to } \\
\text { Converge } \\
\text { (Seconds) }\end{array}$ \\
\hline WFP & n/a & 3379 & 166 & N/A $^{\dagger}$ & N/A $^{\dagger}$ \\
\hline PVRP & n/a & 2509 & 167 & N/A $^{\dagger}$ & N/A $^{\dagger}$ \\
\hline AVRP & 7 & 1052 & 221 & 23.3 & 0.3 \\
\hline AVRP-BLS & 7 & 1165 & 34 & 1.6 & 4.4 \\
\hline Switching & 1 & 1466 & 12 & $9.5^{\ddagger}$ & 0.1 \\
\hline
\end{tabular}

$\dagger$ WFP and PVRP do not actively compensate for shadows.

$\ddagger$ We evaluated a CPU only version of the switching algorithm so that the FPS numbers are an accurate representation of the relative computational complexity of the active algorithms. The GPU version of the switching algorithm runs at $85 \mathrm{fps}$, limited by the refresh rate of our projectors.

the motion of a dynamic occluder in our lab with sufficient precision to ensure repeatability.

As each algorithm reacted to the occluder (WFP and PVRP took no action) the sum squared difference in pixel values of the camera image from the reference image was recorded on each iteration of the algorithm. A second camera recorded the relative light levels falling on the occluder. An overview of the results are presented in Figure 15 and Table I.

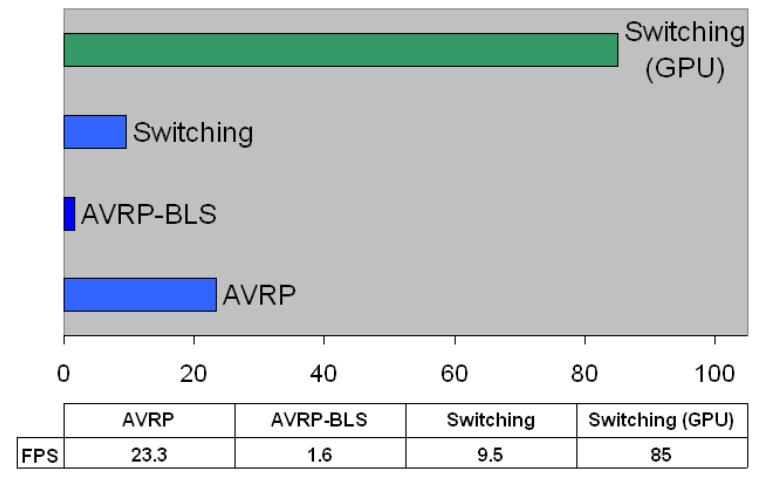

Fig. 13. Speed of Active Systems Relative speed in frames per second (FPS) of the active systems. The GPU implementation of the switching algorithm is provided for comparison on the right. The 85 fps speed is limited by the output speed of the projectors, the actual update rate on the GPU is $101 \mathrm{fps}$.

\section{A. Experimental Setup}

Each algorithm was run on a dual processor Intel $^{\circledR}$ Pentium 4 Xeon $^{\mathrm{TM}}$ 2.2Ghz Dell Precision workstation with 2 GB of RAM. An nVidia GeForceFX 5800 Ultra graphics card on an AGP $4 \times$ bus drove two Hitachi CP-SX 5600 LCOS projectors. The projectors were mounted $430 \mathrm{~cm}$ apart on 


\begin{tabular}{|c|c|c|c|c|c|c|}
\hline & $\begin{array}{l}\text { Effects } \\
\text { on } \\
\text { shadows }\end{array}$ & $\begin{array}{c}\text { Effects } \\
\text { on blinding } \\
\text { light }\end{array}$ & $\begin{array}{l}\text { 2nd } \\
\text { projector } \\
\text { required }\end{array}$ & Camera & $\begin{array}{c}\text { Camera } \\
\text { requires } \\
\text { unoccluded } \\
\text { view }\end{array}$ & $\begin{array}{c}\text { IR } \\
\text { filter or } \\
\text { dedicated } \\
\text { IR camera }\end{array}$ \\
\hline WFP & $\begin{array}{c}\text { moves shadows } \\
\text { aside }\end{array}$ & none & & & & \\
\hline PVRP & $\begin{array}{l}\text { reduces } \\
\text { shadows }\end{array}$ & none & & & & \\
\hline AVRP & $\begin{array}{c}\text { fills in } \\
\text { shadows }\end{array}$ & none & & & & \\
\hline AVRP-BLS & $\begin{array}{l}\text { fills in } \\
\text { shadows }\end{array}$ & $\begin{array}{c}\text { removes } \\
\text { blinding light }\end{array}$ & & & & \\
\hline Switching & $\begin{array}{l}\text { fills in } \\
\text { shadows }\end{array}$ & $\begin{array}{c}\text { removes } \\
\text { blinding light }\end{array}$ & & & & \\
\hline
\end{tabular}

Fig. 14. System Features \& Hardware Requirements

a bar $360 \mathrm{~cm}$ from the display surface, $240 \mathrm{~cm}$ above the floor. The display surface was $181 \mathrm{~cm}$ wide and $130 \mathrm{~cm}$ high, mounted so that it's bottom was $63 \mathrm{~cm}$ from the floor. Each projector was $34^{\circ}$ off of the projection surface's normal, giving a total angular separation of $68^{\circ}$ between the projectors.

A Sony N50 3CCD progressive scan camera was used to measure the sum squared distance (SSD) pixel error seen with respect to a reference image captured before the occluder was introduced. Each algorithm was initially started with no occlusions, and allowed to initialize normally. The system was then paused, and a static occluder was introduced, partially blocking the beam of the first projector. The occluder was a $40.6 \mathrm{~cm}$ wide by $50.8 \mathrm{~cm}$ high white painters canvas, mounted on a tripod $150 \mathrm{~cm}$ from the screen.

After the occluder was introduced, the system was re-started. To the algorithms, this gave the appearance of an instantly appearing occluder which blocked approximately 30 percent of one projector. In the graphs, the occluder appears in frame five.

At this point, the algorithms were allowed to run normally until they had stabilized.

In the simple cases of warped front projection and passive virtual rear projection, the system performed no compensation, and the light on the occluder and errors in the displayed image are immediately stable. As you can see from Table I (SSD Error) and the graphs in Figure 15 passive virtual rear projection improved the image quality over that achieved by a single projector solution (Warped Front Projection) despite taking no implicit compensatory action.

Active virtual rear projection (AVRP), which attempts only to minimize the error of the displayed image, required seven iterations to converge, or 0.3 seconds in real time. After convergence, the SSD error was effectively the same as before the occluder was introduced, although the light cast on the occluder was more than in the non-active cases. This is due to the fact that the AVRP algorithm increases light output from both projectors when attempting to correct a shadow, leading to increased light cast on the occluder.

Active virtual rear projection with blinding light suppression (AVRP-BLS) also took seven iterations to converge, but due to the increased processing required by this algorithm, this equated to 4.4 seconds in real time. The benefit of the additional computational time is shown in the amount of light remaining on the occluder, which as reduced significantly when compared to the previously described algorithms.

The switched VRP system is able to compensate immediately after detecting the occluder (one iteration, or 0.1 seconds). Because it does not employ a feedback loop, the SSD error after compensation is larger than in the AVRP or AVRP-BLS case, but the subjective image quality is good. Occluder light suppression is excellent, with the amount of light cast on the occluder lower than any other algorithm. 
Additionally, it has the fastest real-time performance of the algorithms discussed.

\section{DISCUSSION}

In this paper, we have discussed five methods for improving the robustness of front projected displays in the face of occlusions and shadows. Figure 14 outlines the system features and hardware requirements of the algorithms discussed. Warped front projection (WFP) is the simplest and cheapest method, which places the projector at an acute angle to the display surface. It requires only a 3D graphics accelerator to warp the output. WFP can also be implemented using specialized optics, such as used by the NEC WT600 [15] or 3M Digital WallDisplay [14]. Compared to front projection, WFP reduces the number of occlusions users experience when working with an interactive surface.

Passive Virtual Rear Projection (PVRP) increases the system cost by requiring a second projector and additional video port on the driving computer. In return for this additional cost, the displayed image is made much more robust due to the redundant illumination. In user studies, PVRP was preferred by users over WFP, virtually eliminated occlusions, and eliminated user shadow coping behavior observed in front projection and warped front projection displays [19].

Active Virtual Rear Projection (AVRP) requires the addition of a camera to provide feedback to the system, and has the drawback of projecting extra light on occluders, illustrated in the Occluder Light column of Table I and the AVRP graph of Figure 15. Its benefit is that unlike PVRP, AVRP can compensate for shadows on the display, reducing the overall error in the projected image.

All of the above methods project light on occluders (AVRP even increases the amount of light projected on occluders) which users find annoying. The Active Virtual Rear Projection with Blinding Light Suppression (AVRP-BLS) algorithm determines when projectors are occluded and reduces light being projected on occluders. The main drawback of this algorithm is that it is much more computationally demanding than AVRP which reduces its real world performance.

Both AVRP and AVRP-BLS assume that the camera has an unoccluded view of the display surface. With good physical placement of the camera, this can be achieved in many situations, but is a serious drawback when the user moves very close to the screen. An even larger drawback is the reliance on pre-shot reference images. Although this drawback can be removed by developing a photometric model to predict how arbitrary graphics should appear when projected [22] the systems are still limited by the unoccluded camera assumption.

The switching VRP algorithm, even when implemented in software, is significantly faster than the AVRP-BLS algorithm (Table I, F.P.S. column), and is easily implementable in the Graphics Processing Unit (GPU) of modern graphics cards, as shown in Section VI-D to achieve performance levels that are bottle-necked by the projector refresh rate (currently $85 \mathrm{~Hz}$ ). The only downside to the switching VRP algorithm is that it does not use a feedback system to regulate the light projected to compensate for shadows. This results in images which, although they may appear seamless to users, may deviate from the original projected image in overall intensity (Table I, SSD Error column).

\section{Conclusion}

We recommend system adopters use warped front projection (WFP) when limited to a single projector or looking to purchase an off-the-shelf solution. When two projectors are available, passive virtual rear projection (PVRP) is easy to implement and provides good robustness in the face of occlusions. The Switching form of virtual rear projection is the best method that makes use of active compensation for shadows due to the ability to implement it in GPUs of commodity graphics cards and its good balance of speed and image quality.

The techniques presented here enable the projection of interactive displays to arbitrary positions in the environment from steerable or manually redeployable projectors. The suppression of blinding light and elimination of shadows on the display allows for displays to be positioned where they are needed, and re-positioned on the fly regardless of the position of potential occluders in the environment.

We intend to continue this work by (1) demonstrating virtual rear projection on steerable projectors, (2) locating projectors with lower latency to improve our overall system performance, and (3) improving the switching algorithm to implement 

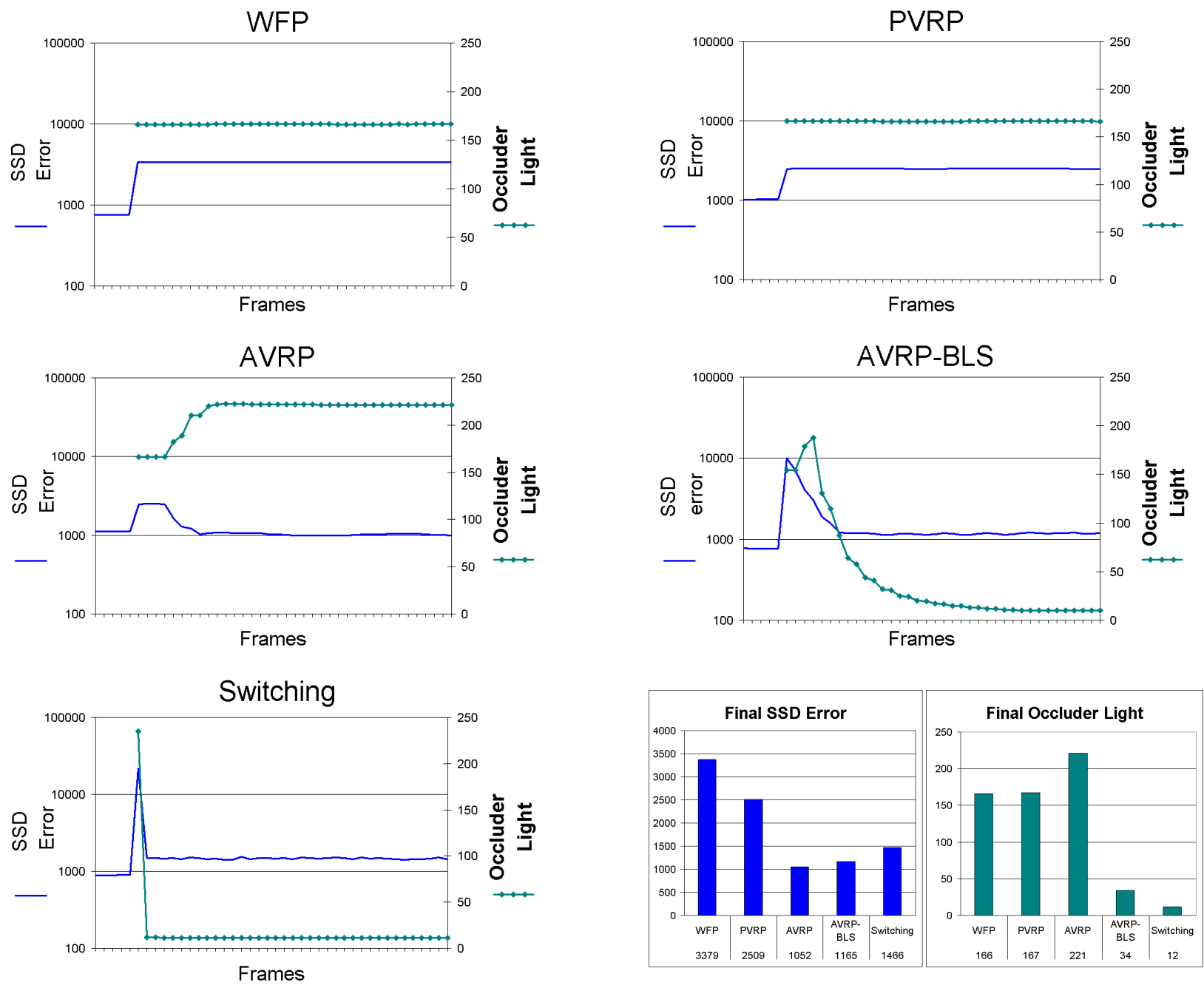

Fig. 15. Top Left: Warped Front Projection Top Right: Passive Virtual Rear Projection Middle Left: Active Virtual Rear Projection Middle Right: Active Virtual Rear Projection with Blinding Light Suppression Bottom Left: Switching VRP Bottom Right: Final SSD and Occluder Light Measures

optical-flow on the GPU to predict where occlusions will occur and eliminate shadows before they are cast.

\section{REFERENCES}

[1] R. Yang, D. Gotz, J. Hensley, H. Towles, and M. Brown, "PixelFlex: A reconfigurable multi-projector display system," in Proceedings of IEEE Visualization, 2001.

[2] M. Hereld, I. Judson, and R. Stevens, "Introduction to building projection-based tiled displays," Computer Graphics and Applications, vol. 20, no. 4, 2000.

[3] K. Li, H. Chen, Y. Chen, D. Clark, P. Cook, S. Daminakis, G. Essl, A. Finkelstein, T. Funkhouser, A. Klein, Z. Liu, E. Praun, R. Samanta, B. Shedd, J. Singh, G. Tzanetakis, and J. Zheng, "Building and using a scalable display wall system," Computer Graphics and Applications, vol. 20, no. 4, 2000.

[4] R. Surati, "A scalable self-calibrating technology for seamless large-scale displays," Ph.D. dissertation, Department of Electrical Engineering and Computer Science, Massachussetts Institute of Technology, 1999.
[5] G. Humphreys and P. Hanrahan, "A distributed graphics system for large tiled displays," in Proceedings of IEEE Visualization, 1999. [Online]. Available: http://graphics.stanford.edu/papers/ mural_design/

[6] R. Raskar, M. Brown, R. Yang, W. Chen, G. Welch, H. Towles, B. Seales, and H. Fuchs, "Multi-projector displays using camera-based registration," in Proceedings of IEEE Visualization, 1999.

[7] S. Elrod, R. Bruce, R. Gold, D. Goldberg, F. Halasz, W. Janssen, D. Lee, K. McCall, E. Pedersen, K. Pier, J. Tang, and B. Welch, "Liveboard: a large interactive display supporting group meetings, presentations, and remote collaboration," in Proceedings of the SIGCHI conference on Human factors in computing systems. ACM Press, 1992, pp. 599-607.

[8] Smart Technologies, "Smartboard." [Online]. Available: http: //www.smarttech.com/products/index.asp

[9] B. Johanson, A. Fox, and T. Winograd, "The interactive workspaces project: Experiences with ubiquitous compu ting rooms." IEEE Pervasive Computing, vol. 1, no. 2, 2002.

[10] P. R. Waier, Ed., Building Construction Cost Data 2002. Robert S. Means Co., 2002.

[11] C. Pinhanez, "The Everywhere display," in Proceedings of 
Ubiquitous Computing, 2001, pp. 315-331.

[12] C. Pinhanez and M. Podlaseck, "To frame or not to frame: The role and design of frameless displays in ubiquitous applications," in Proceedings of Ubiquitous Computing, 2005, pp. 340-357.

[13] M. Flagg and J. M. Rehg, "Oil painting assistance using projected light: Bridging the gap between digital and physical art," GVU Center, Georgia Institute of Technology, Tech. Rep. GIT-GVU-05-35, March 2005.

[14] 3M, "Digital walldisplay." [Online]. Available: http://www.3m. com/meetings/product_catalog/digitalwalldisplay/index.jhtml

[15] NEC, "Wt-600." [Online]. Available: http://www.nec-pj.com/ products/wt/

[16] R. Sukthankar, R. Stockton, and M. Mullin, "Smarter presentations: Exploiting homography in camera-projector systems," in Proceedings of International Conference on Computer Vision, 2001.

[17] R. Hartley and A. Zisserman, Multiple View Geometry in Computer Vision. New York, NY: Cambridge University Press, 2000.

[18] D. Ballard and C. Brown, Computer Vision. Prentice-Hall, 1982.

[19] J. Summet, G. D. Abowd, G. M. Corso, , and J. M. Rehg, "Virtual rear projection: Do shadows matter?" in CHI'05 Extended Abstracts, 2005, pp. 1997-2000. [Online]. Available: http://www.cc.gatech.edu/ summetj/papers/sp408-summet.pdf

[20] S. Nayar, H. Peri, M. Grossberg, and P. Belhumeur, "A projection system with radiometric compensation for screen imperfections," in First IEEE International Workshop on ProjectorCamera Systems (PR OCAMS-2003), 2003.

[21] T. J. Cham, J. M. Rehg, R. Sukthankar, and G. Sukthankar, "Shadow elimination and occluder light suppression for multi-projector displays." in Proceedings of Computer Vision and Pattern Recognition, 2003, pp. 513-520. [Online]. Available: http://www-2.cs.cmu.edu/ rahuls/pub/ cvpr2003-shadow-rahuls.pdf

[22] C. Jaynes, S. Webb, R. M. Steele, M. Brown, and W. B. Seales, "Dynamic shadow removal from front projection displays," in Proceedings of the conference on Visualization 2001. IEEE Press, 2001, pp. 175-182.

[23] A. Majumder, "Properties of color variation across a multiprojector display," in SID Eurodisplay, 2002.

[24] A. Majuder and R. Stevens, "Effects of projector parameters on color variation in projection based displays." Department of Computer Science, University of North Carolina at Chapel Hill, Tech. Rep. 02-022, 2002.

[25] A. Majumder and R. Stevens, "LAM: Luminance attenuation map for photometric uniformity in projection based displays," in ACM Virtual Reality and Software Technology, 2002. [Online]. Available: citeseer.ist.psu.edu/majumder02lam.html

[26] W. F. Engel, Shaderx2: Shader Programming Tips \& Tricks With Directx 9. Wordware Publishing, 2003, ch. Advanced Image Processing with DirectX 9 Pixel Shaders.

[27] H. Chen, R. Sukthankar, G. Wallace, and K. Li, "Scalable alignment of large-format multi-projector displays using camera homography trees," in Proceedings of Visualization 2002, 2002, pp. 339-346.

[28] D. S. Tan and R. Pausch, "Pre-emptive shadows: Eliminating the blinding light from projectors," in CHI'O2 extended abstracts, 2002, pp. 682-683.

[29] R. Sukthankar, T. J. Cham, and G. Sukthankar, "Dynamic shadow elimination for multi-projector displays," in Proceedings of CVPR, 2001, pp. 151-157. [Online]. Available: http://www-2.cs.cmu.edu/ rahuls/Research/Projector/\#shadow

[30] C. Jaynes, S. Webb, and R. M. Steele, "Camera-based detec- tion and removal of shadows from interactive multiprojector displays," IEEE Transactions on Visualization and Computer Graphics (TVCG), vol. 10, no. 3, May/June 2004.

[31] A. Majumder and R. Stevens, "Color nonuniformity in projection-based displays: Analysis and solutions," IEEE Transactions on Visualization and Computer Graphics, vol. 10, no. 2, pp. 177-188, 2004. 